\title{
HAL QCD method and Nucleon-Omega interaction with physical quark masses
}

\section{Takumi Iritani*}

Theoretical Research Division, Nishina Center, RIKEN, Wako 351-0198, Japan

E-mail: takumi.iritani@riken.jp

\section{for HAL QCD Collaboration}

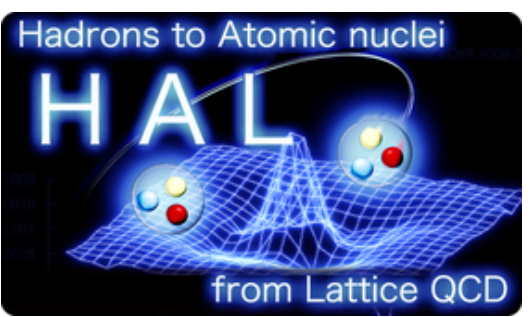

In lattice QCD, both direct method and HAL QCD method are used to investigate the two-baryon systems. We show that due to the contamination of the scattering excited states, it is challenging to measure the eigenenergy from the temporal correlation in the direct method, while the HAL QCD method can extract the information of the interaction from both scattering states and ground state by using the spatial correlation. We examine the systematic uncertainty of the derivative expansion in the HAL QCD method, which is found to be well under control at the low energies. By using the time-dependent HAL QCD method, we study the nucleon $(N)$-Omega $(\Omega)$ system in the ${ }^{5} \mathrm{~S}_{2}$ channel with almost physical quark masses at $m_{\pi} \simeq 146 \mathrm{MeV}$. We find the interaction is attractive at all distances, which produces a quasi-bound state with the binding energy $1.54(0.30)\left({ }_{-0.10}^{+0.04}\right) \mathrm{MeV}$. We also consider the extra Coulomb interaction in the $p \Omega^{-}\left({ }^{5} \mathrm{~S}_{2}\right)$ system, whose binding energy becomes $2.46(0.34)\left({ }_{-0.01}^{+0.04}\right) \mathrm{MeV} . N \Omega\left({ }^{5} \mathrm{~S}_{2}\right)$ dibaryon could be searched through two-particle correlations in the heavy ion collision experiments.

The 36th Annual International Symposium on Lattice Field Theory - LATTICE2018

22-28 July, 2018

Michigan State University, East Lansing, Michigan, USA.

\footnotetext{
* Speaker.
} 


\section{Introduction}

The search for the dibaryons is one of the long-standing problems in hadron physics. The most famous candidate is the $H$-dibaryon(uuddss), which is predicted by the MIT bag model in 1977 [1]. Recently, the model-independent studies of the $H$-dibaryon are reported from lattice QCD calculations $[2,3,4]$. Due to the absence of the Pauli blocking effect, another promising candidate for the dibaryon is $N \Omega$ (uudsss or $u d d s s s$, which is claimed to be bound from the studies based on the phenomenological models $[5,6,7,8,9,10,11]$. This dibaryon is also reported from the (2+1)-flavor lattice QCD study for the heavier pion mass at $m_{\pi} \simeq 875 \mathrm{MeV}$ [12].

In order to clarify the existence of the dibaryon, the reliable calculation from lattice QCD is important. However, in the previous studies for the two-baryon systems for heavier pion masses, inconsistent conclusions are reported from two lattice QCD approaches [13, 14, 15]. In this work, we discuss the fundamental difficulty in the two-baryon systems from the direct method by using the temporal correlation due to the contamination of the scattering states, while the HAL QCD method is free from such an issue by using the spatial correlation $[16,17,18]$. We also show the systematic uncertainty of the derivative expansion in the HAL QCD method is well under control. By using the HAL QCD method, we discuss the $N \Omega$ system in the ${ }^{5} \mathrm{~S}_{2}$ channel with almost physical quark masses at $m_{\pi} \simeq 146 \mathrm{MeV}$ [19].

\section{Two-Baryon Systems from Lattice QCD}

\subsection{Direct method and the pseudo plateaux problem}

In the direct method, the energy eigenvalue of the two-baryon system is extracted from the temporal correlation. In practical lattice QCD calculations, the energy shift $\Delta E_{L}=E_{\mathrm{BB}}^{L}-2 m_{B}$ at a finite box with the spatial extension $L$ is obtained by the plateau of the effective energy shift $\Delta E_{\mathrm{BB}}^{\mathrm{eff}}(t)$, which is defined by

$$
\Delta E_{\mathrm{BB}}^{\mathrm{eff}}(t) \equiv \frac{1}{a} \log \frac{R_{\mathrm{BB}}(t)}{R_{\mathrm{BB}}(t+a)}
$$

where $R_{\mathrm{BB}}(t) \equiv C_{\mathrm{BB}}(t) /\left\{C_{\mathrm{B}}(t)\right\}^{2}$ with the two-baryon (single baryon) correlator $C_{\mathrm{BB}}(t)\left(C_{\mathrm{B}}(t)\right)$ and a lattice spacing $a$.

One of the problems in the multi-baryon systems is the signal to noise ratio, which becomes exponentially worse as $S(t) / N(t) \sim \exp \left[-A\left(m_{B}-(3 / 2) m_{M}\right) t\right]$, where $A$ is the baryon number, $m_{B}$ and $m_{M}$ are the baryon and meson masses, respectively. Furthermore, the contamination of the elastic excited states can be a severe problem. The energy gap of the scattering states is proportional to $\mathscr{O}\left(1 / L^{2}\right)$, which is much smaller than the inelastic gap $\mathscr{O}\left(\Lambda_{\mathrm{QCD}}\right)$. It means the ground state saturation of the two-baryon system requires a large Euclidean time than that of the single particle system.

To demonstrate this point [17], we consider a mock-up correlator as

$$
R(t)=b_{0} e^{-\Delta E_{\mathrm{BB}} t}+b_{1} e^{-\left(\delta E_{\mathrm{el}}+\Delta E_{\mathrm{BB}}\right) t}+c_{0} e^{-\left(\delta E_{\mathrm{inel}}+\Delta E_{\mathrm{BB}}\right) t},
$$

with the energy shift $\Delta E_{\mathrm{BB}}=E_{\mathrm{BB}}-2 m_{\mathrm{B}}$, and the energy gap of the elastic(inelastic) excited state $\delta E_{\text {el }}\left(\delta E_{\text {inel }}\right)$. Here, we adopt $\delta E_{\text {el }}=50 \mathrm{MeV}$ and $\delta E_{\text {inel }}=500 \mathrm{MeV}$, which are typical scales of 
the current lattice QCD simulations. Fig. 1 (Left) shows $\Delta E_{\mathrm{BB}}^{\mathrm{eff}}(t)-\Delta E_{\mathrm{BB}}$ for $c_{0} / b_{0}=0.01$ and $b_{1} / b_{0}=0.1,0,-0.1$. The inelastic state becomes negligible around $1 \mathrm{fm}$, while $\mathscr{O}(10) \mathrm{fm}$ of the Euclidean time is required for the ground state saturation with the elastic excited state.

The effective energy shifts with fluctuations are shown in Fig. 1 (Right). There are plateau-like structures around $t \sim 1 \mathrm{fm}$. However, these are incorrect signals except black circles $\left(b_{1} / b_{0}=0\right)$. It shows the plateau-like behavior cannot guarantee the ground state saturation at all.
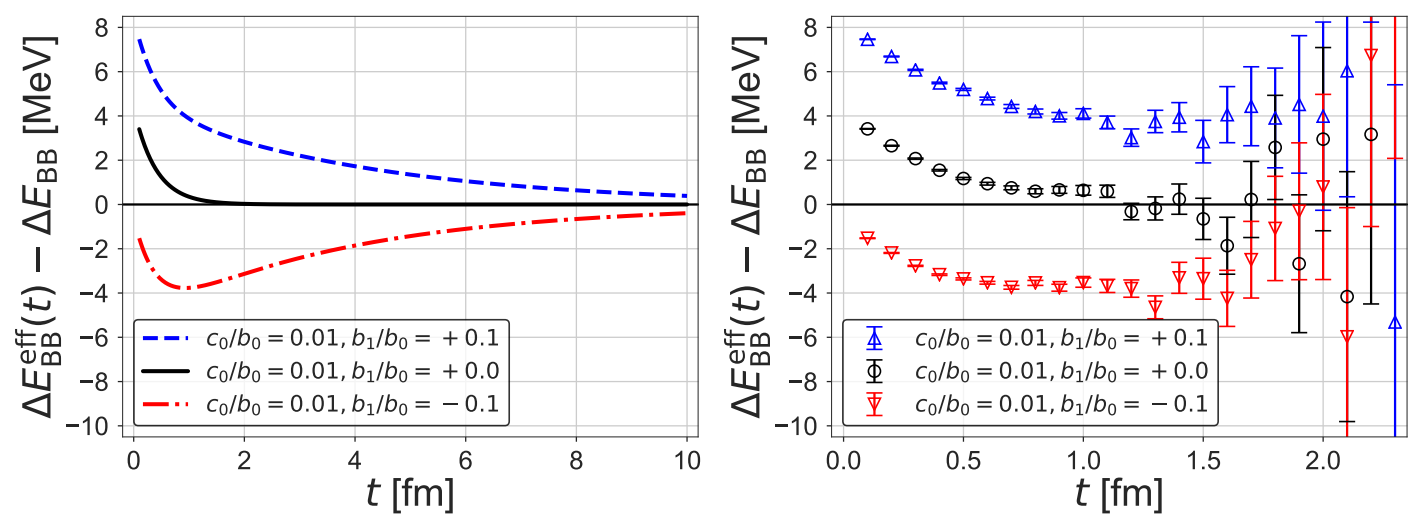

Figure 1: (Left) The effective energy shift of the mock-up data. (Right) A mock-up data with fluctuations.

These pseudo plateaux cast a question on the previous studies of two-nucleon systems by the direct method [13], which depend on the naive plateaux fitting without employing a variational method. In Ref. [20, 21], we discuss the normality of these results based on the Lüscher's finite volume formula [22], and clarify all of them show anomalous behaviors, for example, the parameters of the effective range expansion are found to be singular, the S-matrix pole has the unphysical residue. These symptoms imply the misidentification of the eigenenergies.

\subsection{HAL QCD method and the convergence of the derivative expansion}

The time-dependent HAL QCD method extracts the information of the interaction by using all scattering states below the inelastic threshold [16]. In this method, the energy-independent and non-local potential $U\left(\vec{r}, \vec{r}^{\prime}\right)$ is given by

$$
\left[-H_{0}-\frac{\partial}{\partial t}+\frac{1}{4 m_{B}} \frac{\partial^{2}}{\partial t^{2}}\right] R(\vec{r}, t)=\int d \overrightarrow{r^{\prime}} U\left(\vec{r}, \vec{r}^{\prime}\right) R\left(\vec{r}^{\prime}, t\right) .
$$

Here the $R$-correlator is defined as

$$
R(\vec{r}, t) \equiv\langle 0| T\left\{B(\vec{x}+\vec{r}, t) B(\vec{x}, t) \overline{\mathscr{J}}(0)|0\rangle /\left\{C_{B}(t)\right\}^{2}=\sum_{n} A_{n} \psi^{W_{n}}(\vec{r}) e^{-\Delta W_{n} t}+\mathscr{O}\left(e^{-\Delta W_{\mathrm{th}} t}\right)\right.
$$

with a source operator $\mathscr{J}$, the Nambu-Bethe-Salpeter wave function $\psi^{W_{n}}(r), \Delta W_{n}=W_{n}-2 m_{B}$ with $n$-th energy eigenvalue $W_{n}$, and the inelastic threshold $\Delta W_{\text {th }}=W_{\text {th }}-2 m_{B}$. For the spinsinglet channel, the potential in the leading order (LO) analysis of the velocity expansion $U\left(\vec{r}, \vec{r}^{\prime}\right)=$ $\sum_{n} V_{n}(\vec{r}) \nabla^{n} \delta\left(\vec{r}-\vec{r}^{\prime}\right)$ is given by

$$
V_{0}^{\mathrm{LO}}(r)=-\frac{H_{0} R(\vec{r}, t)}{R(\vec{r}, t)}-\frac{(\partial / \partial t) R(\vec{r}, t)}{R(\vec{r}, t)}+\frac{1}{4 m_{B}} \frac{\left(\partial^{2} / \partial t^{2}\right) R(\vec{r}, t)}{R(\vec{r}, t)} .
$$


To confirm its convergence, we consider the next-to-next-to-leading order $\left(\mathrm{N}^{2} \mathrm{LO}\right)$ analysis as $U\left(\vec{r}, \vec{r}^{\prime}\right) \simeq\left\{V_{0}^{\mathrm{N}^{2} \mathrm{LO}}(r)+V_{2}^{\mathrm{N}^{2} \mathrm{LO}}(r) \nabla^{2}\right\} \delta\left(\vec{r}-\vec{r}^{\prime}\right)$. The relation among these potentials is given by

$$
V_{0}^{\mathrm{LO}}(r)=V_{0}^{\mathrm{N}^{2} \mathrm{LO}}(r)+V_{2}^{\mathrm{N}^{2} \mathrm{LO}}(r) \frac{\nabla^{2} R(\vec{r}, t)}{R(\vec{r}, t)},
$$

which means the $\mathrm{N}^{2} \mathrm{LO}$ correction in $V_{0}^{\mathrm{LO}}$ depends on both $V_{2}^{\mathrm{N}^{2} \mathrm{LO}}$ and the $R$-correlator.

Fig. 2 (Left) shows $V_{0}$ potential of $\Xi \Xi\left({ }^{1} \mathrm{~S}_{0}\right)$ at $m_{\pi}=510 \mathrm{MeV}$ from the $\mathrm{LO}$ analysis by using wall-type quark source and the $\mathrm{N}^{2} \mathrm{LO}$ analysis. The $\mathrm{S}$-wave scattering phase shifts from the LO and the $\mathrm{N}^{2} \mathrm{LO}$ analyses are shown in Fig. 2 (Right). As shown in these figures, the LO analysis from the wall source works well at the low energies, while the $\mathrm{N}^{2} \mathrm{LO}$ correction appears at high energies. These results imply that the systematic uncertainties of the derivative expansion are well under control at the low energies [18].
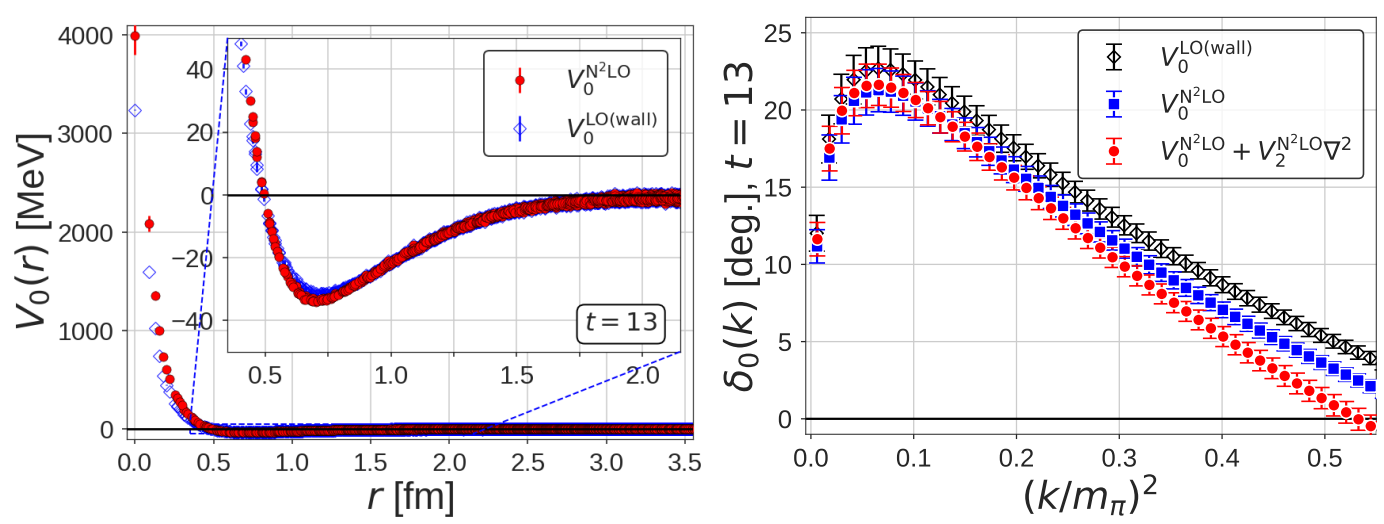

Figure 2: (Left) The leading order potential of $\Xi \Xi\left({ }^{1} \mathrm{~S}_{0}\right)$ at $m_{\pi}=510 \mathrm{MeV}$. (Right) The S-wave scattering phase shift as a function of $\left(k / m_{\pi}\right)^{2}$ from $V_{0}^{\mathrm{LO}(\text { wall })}, V_{0}^{\mathrm{N}^{2} \mathrm{LO}}$ and $V_{0}^{\mathrm{N}^{2} \mathrm{LO}}+V_{2}^{\mathrm{N}^{2} \mathrm{LO}} \nabla^{2}$. Both figures are taken from [18].

\section{3. $N \Omega$ system at almost physical quark masses}

Next we apply the HAL QCD method to the $N \Omega$ system in the ${ }^{5} \mathrm{~S}_{2}$ channel at almost physical masses $\left(m_{\pi} \simeq 146 \mathrm{MeV}\right.$ and $\left.m_{K} \simeq 525 \mathrm{MeV}\right)$ [19]. The lattice volume is $96^{4}$ with a lattice cutoff $a^{-1}=2.333 \mathrm{GeV}$ [23]. We employ the wall-type quark source with the Coulomb gauge fixing. Total number of the measurements is $119,232,{ }^{1}$ and the statistical errors are estimated by the jack-knife sampling. The masses of a nucleon and $\Omega$ are 954.7(2.7) $\mathrm{MeV}$ and 1711.5(1.0) $\mathrm{MeV}$, respectively, which are slightly heavier than the physical values.

In this work, we consider a single channel potential of the $N \Omega\left({ }^{5} \mathrm{~S}_{2}\right)$ in the LO analysis of the derivative expansion. Strictly speaking, $N \Omega\left({ }^{5} \mathrm{~S}_{2}\right)$ decays into the D-wave states of $\Sigma \Xi$ and $\Lambda \Xi$. We assume the coupling to these states is kinematically suppressed ${ }^{2}$.

\footnotetext{
${ }^{1}$ The statistics are slightly improved from the result at the conference.

${ }^{2}$ The effect of these coupling channels is found to be small from the phenomenological study in Ref. [11], nevertheless, the coupled channel analysis would be required to confirm this assumption in the future [3].
} 
Fig. 3 (Left) shows the central potential $V_{\mathrm{C}}(r)$ at $t / a=11-14$. These results are consistent with each other within the statistical errors. It indicates the smallness of the coupling to the Dwave octet-octet systems. $V_{\mathrm{C}}(r)$ is attractive in all distances, which is qualitatively the same as the previous study at heavier quark masses [12].
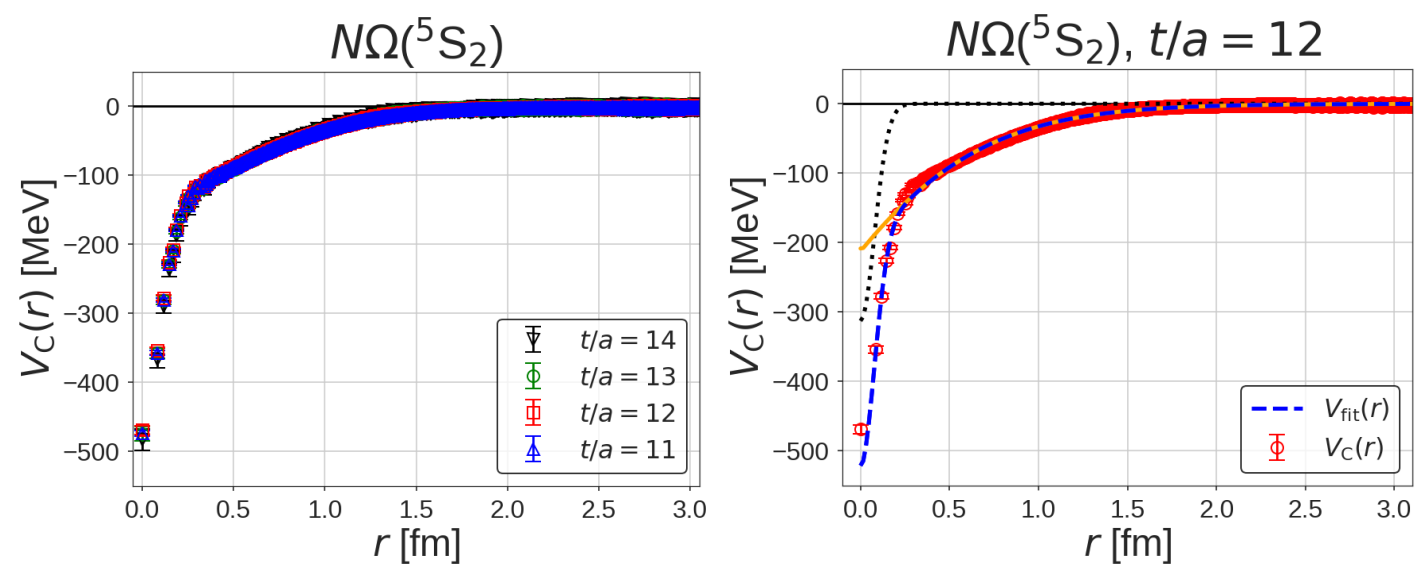

Figure 3: (Left) The central potential $V_{c}(r)$ of the $N \Omega\left({ }^{5} \mathrm{~S}_{2}\right)$ at $t / a=11-14$. (Right) The result of the fitting of $V_{\mathrm{C}}(r)$ (red circles) by using Eq. (3.1) at $t / a=12$ (blue dashed line). The black dotted and the orange solid lines correspond to the first and the second term in Eq. (3.1), respectively. Both figures are taken from [19].

In order to calculate the scattering phase shifts and the binding energy, we fit the potential by using Gaussian $+(\text { Yukawa })^{2}$ with a form factor [12] as

$$
V_{\text {fit }}(r)=c_{0} e^{-c_{1} r^{2}}+c_{2}\left(1-e^{-c_{3} r^{2}}\right)^{n}\left(\frac{e^{-m_{\pi} r}}{r}\right)^{2} .
$$

We find that $n=1$ with $m_{\pi}=146 \mathrm{MeV}$ works well as shown in Fig. 3 (Right). The details of the analyses and parameters are summarized in Ref. [19].

Fig. 4 (Left) shows the S-wave scattering phase shifts $\delta_{0}$ as a function of the kinetic energy. These results are consistent with each other within the errors from $t / a=11$ to 14 , and it approaches to $180^{\circ}$ at $k \rightarrow 0$. As shown in Fig. 4 (Right), the scattering length $a_{0} \equiv-\lim _{k \rightarrow 0} \tan \delta_{0}(k) / k$ becomes positive. These results mean the formation of a quasi-bound state of $N \Omega$ in the ${ }^{5} \mathrm{~S}_{2}$ channel.

The effective range expansion up to the NLO is given by $k \cot \delta_{0}=-\frac{1}{a_{0}}+\frac{1}{2} r_{\text {eff }} k^{2}$, where $r_{\text {eff }}$ is the effective range. These parameters are found to be $a_{0}=5.30(0.44)\left({ }_{-0.01}^{+0.16}\right) \mathrm{fm}$ and $r_{\text {eff }}=$ $1.26(0.01)\left({ }_{-0.01}^{+0.02}\right) \mathrm{fm}$, where the central values and the statistical errors are obtained at $t / a=12$, and the systematic errors in the second parentheses are estimated from the central values at $t / a=11$, 13 and 14. The smallness of $r_{\text {eff }} / a_{0}$ suggests this dibaryon system is close to the unitary limit.

The binding energy $B$ and the root mean square distance $\sqrt{\left\langle r^{2}\right\rangle}$ are found to be $B=1.54(0.30)\left({ }_{-0.10}^{+0.04}\right) \mathrm{MeV}$ and $\sqrt{\left\langle r^{2}\right\rangle}=3.77(0.31)\left({ }_{-0.01}^{+0.11}\right) \mathrm{fm}$. The size of the $N \Omega\left({ }^{5} \mathrm{~S}_{2}\right)$ bound state is comparable to its scattering length, which implies this system is a loosely bound state like deuteron from the experiments and $\Omega \Omega\left({ }^{1} \mathrm{~S}_{0}\right)$ from the lattice QCD calculation [24].

Finally, we consider the $p \Omega^{-}\left({ }^{5} \mathrm{~S}_{2}\right)$ system with the Coulomb interaction. By using $V_{\text {fit }}(r)-$ $\alpha / r$ with $\alpha \equiv e^{2} /(4 \pi)=1 / 137.036$, we obtain $B_{p \Omega^{-}}=2.46(0.34)\left(_{-0.11}^{+0.04}\right) \mathrm{MeV}$ and ${\sqrt{\left\langle r^{2}\right\rangle}}_{p \Omega^{-}}=$ 

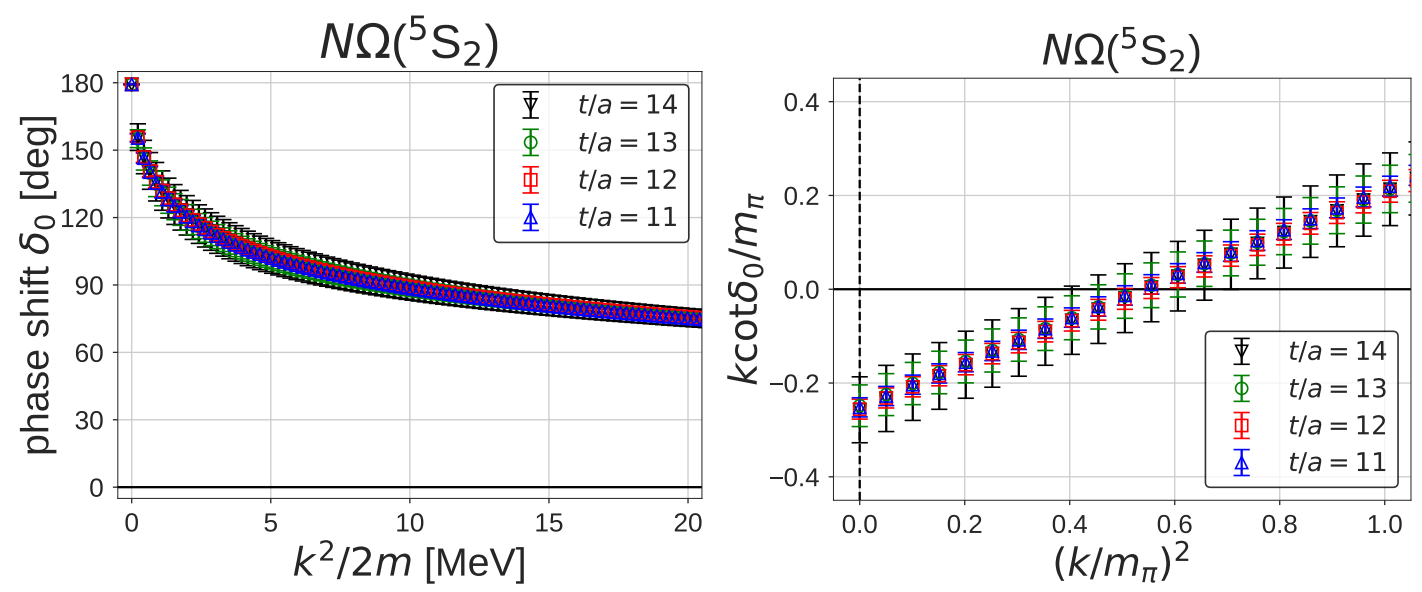

Figure 4: (Left) The S-wave scattering phase shifts $\delta_{0}$ as a function of the kinetic energy from $t / a=11-14$. (Right) $k \cot \delta_{0} / m_{\pi}$ as a function of $\left(k / m_{\pi}\right)^{2}$ from $t / a=11-14$. Both figures are taken from [19].

$3.24(0.19)\left({ }_{-0.00}^{+0.06}\right) \mathrm{fm}$. Due to the additional attractive interaction, $p \Omega^{-}\left({ }^{5} \mathrm{~S}_{2}\right)$ dibaryon becomes slightly deeper and compact bound system than $n \Omega^{-}\left({ }^{5} S_{2}\right)$ dibaryon.

\section{Summary}

In this paper, we have discussed the two-baryon systems from lattice QCD by using the direct method and the HAL QCD method. The contamination of the scattering states can cause serious uncertainties in the eigenenergies from the simple plateau fitting in the direct method. Therefore, the variational method is mandatory to extract reliable eigenenergies. On the other hand, the HAL QCD method is free from such a problem, and systematic uncertainty in the derivative expansion has been shown to be under control.

By using the HAL QCD method, we have studied the $N \Omega\left({ }^{5} \mathrm{~S}_{2}\right)$ system with almost physical quark masses. We have found a strong attractive potential in all distances, which produces a dibaryon state. This state can be searched by the two-particle correlation at the heavy ion collisions [25]. Recently, $N \Omega$ correlation is reported by the STAR Collaboration at RHIC [26], and updated theoretical analyses by using the HAL QCD potentials near the physical point will be reported elsewhere [27].

\section{Acknowledgements}

The lattice QCD calculations have been performed on Blue Gene/Q at KEK (Nos. 12/13-19, 13/14-22, 14/15-21, 15/16-12), HA-PACS at University of Tsukuba (Nos. 13a-23, 14a-20) and K computer at AICS (hp120281, hp130023, hp140209, hp150085, hp150223, hp150262, hp160093, hp160211, hp170230), HOKUSAI FX100 computer at RIKEN, Wako (G15023, G16030, G17002). This research was supported by MEXT as "Priority Issue on Post-K computer" (Elucidation of the Fundamental Laws and Evolution of the Universe) and JICFuS. 


\section{References}

[1] R. L. Jaffe, Phys. Rev. Lett. 38, 195 (1977) Erratum: [Phys. Rev. Lett. 38, 617 (1977)]. 10.1103/PhysRevLett.38.195

[2] T. Inoue et al. [HAL QCD Collaboration], Nucl. Phys. A 881, 28 (2012) [arXiv:1112.5926 [hep-lat]].

[3] K. Sasaki et al. [HAL QCD Collaboration], EPJ Web Conf. 175, 05010 (2018).

[4] A. Francis, J. R. Green, P. M. Junnarkar, C. Miao, T. D. Rae and H. Wittig, arXiv:1805.03966 [hep-lat].

[5] J. T. Goldman, K. Maltman, G. J. Stephenson, Jr., K. E. Schmidt and F. Wang, Phys. Rev. Lett. 59, 627 (1987).

[6] M. Oka, Phys. Rev. D 38, 298 (1988).

[7] Q. B. Li and P. N. Shen, Eur. Phys. J. A 8, 417 (2000) 10.1007/s10050-000-5080-y [nucl-th/9910060].

[8] H. r. Pang, J. l. Ping, F. Wang, J. T. Goldman and E. g. Zhao, Phys. Rev. C 69, 065207 (2004) [nucl-th/0306043].

[9] X. Zhu, H. Huang, J. Ping and F. Wang, Phys. Rev. C 92, no. 3, 035210 (2015) [arXiv:1507.05851 [hep-ph]].

[10] H. Huang, J. Ping and F. Wang, Phys. Rev. C 92, 065202 (2015) [arXiv:1507.07124 [hep-ph]].

[11] T. Sekihara, Y. Kamiya and T. Hyodo, Phys. Rev. C 98, 015205 (2018) [arXiv:1805.04024 [hep-ph]].

[12] F. Etminan et al. [HAL QCD Collaboration], Nucl. Phys. A 928, 89 (2014) [arXiv:1403.7284 [hep-lat]].

[13] Z. Davoudi, EPJ Web Conf. 175, 01022 (2018) [arXiv:1711.02020 [hep-lat]], and the references therein.

[14] N. Ishii, S. Aoki and T. Hatsuda, Phys. Rev. Lett. 99 (2007) 022001 [nucl-th/0611096].

[15] S. Aoki et al. [HAL QCD Collaboration], PTEP 2012 (2012) 01A105 [arXiv:1206.5088 [hep-lat]].

[16] N. Ishii et al. [HAL QCD Collaboration], Phys. Lett. B 712 (2012) 437 [arXiv:1203.3642 [hep-lat]].

[17] T. Iritani et al., JHEP 1610 (2016) 101 [arXiv:1607.06371 [hep-lat]].

[18] T. Iritani et al. [HAL QCD Collaboration], arXiv:1805.02365 [hep-lat].

[19] T. Iritani et al., arXiv:1810.03416 [hep-lat].

[20] T. Iritani et al., Phys. Rev. D 96, no. 3, 034521 (2017) [arXiv:1703.07210 [hep-lat]].

[21] S. Aoki, T. Doi and T. Iritani, EPJ Web Conf. 175, 05006 (2018) [arXiv:1707.08800 [hep-lat]].

[22] M. Lüscher, Nucl. Phys. B 354, 531 (1991).

[23] K.-I. Ishikawa et al. [PACS Collaboration], PoS LATTICE 2015, 075 (2016) [arXiv:1511.09222 [hep-lat]].

[24] S. Gongyo et al., Phys. Rev. Lett. 120, no. 21, 212001 (2018) [arXiv:1709.00654 [hep-lat]].

[25] K. Morita, A. Ohnishi, F. Etminan and T. Hatsuda, Phys. Rev. C 94, no. 3, 031901 (2016) [arXiv:1605.06765 [hep-ph]].

[26] J. Adam et al. [STAR Collaboration], arXiv:1808.02511 [hep-ex].

[27] K. Morita, S. Gongyo, T. Hatsuda, T. Iritani, A. Ohnishi, and K. Sasaki, in preparation. 